\title{
Numerical Investigation on Heat and Mass transfer through a porous medium in the presence of MHD and Chemical Reaction
}

\author{
R.Kavitha $^{a}$, Ajay Prakash Pasupulla ${ }^{\mathrm{b}}$ and P. Panneerselvam ${ }^{\mathrm{c}}$ \\ aDepartment of Mathematics, Rajalakshmi Institute of Technology, Chennai - 600124 \\ B Professor, Oral and Maxillo Facial Pathologist, Department, School of Medicine, Wochemo University, Hosanna, Ethiopia, \\ East frica \\ cDepartment of Mathematics, Sona College of Arts and Science, Salem-5
}

Article History: Received: 11 January 2021; Accepted: 27 February 2021; Published online: 5 April 2021

\begin{abstract}
An attempt has been made to investigate the heat and mass transfer through a porous medium in the presence of Magnetohydrodynamic flow and chemical reaction. On the basis of certain assumptions, the momentum, energy and concentration equations are obtained. These governing equations are transformed into ordinary differential equations using self-suitable transformations and these resulting equations are solved numerically using shooting procedure with fourth order Runge-Kutta Method. The effect of various non- dimensional parameters like magnetic parameter (M), Schmidt number (Sc), Chemical reaction $(\mathrm{Kr})$ are discussed with the help of graphs.
\end{abstract}

Keywords: MHD, Heat transfer, Porous medium, steady flow, Chemical reaction

\section{Introduction}

The Magnetohydrodynamics flow plays a vital role in engineering fields such as heat exchanger devices, petroleum reservoirs, chemical catalytic reactors and processes, geothermal and geophysical engineering, aerodynamic engineering and others. Effect of chemical reaction also occurs in many branches of science and engineering. Chemical processes have numerous industrial applications such as manufacturing of glassware, food processing, filtration and purification process in chemical engineering, etc., The effect of heat and mass transfer in the presence of MHD flow with chemical reaction has many applications in design of chemical processing equipment's, formation and dispersion of flog, food processing and cooling towers.

Santosh Chaudhary and Pradeep Kumar [9] has extended the work of Andersson[1], who has studied the effects of slip boundary layer stagnation point flow and heat transfer towards a shrinking sheet. Santhosh $e t$ al.[9] has discussed viscous incompressible, electrically conducting fluid near a stagnation point past a shrinking sheet with slip in the presence of a magnetic field. Bhattacharyya and Pop [2] studied MHD boundary layer flow due to an exponentially shrinking sheet. Bhattacharyya and Layek[3] investigated the MHD boundary layer flow over a penetrable stretching sheet with suction/blowing and chemical reaction. The Authors [4] have extended their work in 2012 and got the similarity solution of MHD boundary layer flow with diffusion and chemical reaction using numerical approach. Kandasamy [5] analysed the radiative heat transfer over a porous convective surface in the presence of magnetic field. Mallikarjuna et al. [6] investigated the numerical approach of an unsteady MHD convective flow on heat and mass transfer of circular cone through porous medium in the presence of chemical reaction. Mohanty et al. [7] depicted the study of unsteady heat and mass transfer characteristics of a viscous incompressible electrically conducting micropolar fluid through porous medium in the presence of viscous dissipation using numerical method.

Ullah et al. [8] studied the mixed convective flow of MHD Casson fluid in the presence of chemical reaction. The authors used the similarity transformations to convert partial differential equations into nonlinear ordinary differential equations, and the resulting equations are solved using Keller-box method. Sarma and Mahanta [10] studied the effect of chemical reaction on three-dimensional mass transfer flow in the presence of magnetic field using perturbation technique.

To the best of authors' knowledge, effect of MHD and Chemical Reaction on a free convective flow with heat transfer and mass concentration is of great importance, have not been reported. This motivated us to carry out the present work.

The aim of this paper is to study the effect of heat and mass transfer through porous medium in the presence of MHD flow and chemical reaction. The governing equations are transformed by a similarity transformation into a system of non-linear ordinary differential equations which are solved numerically by fourth order RungeKutta Method using shooting procedure. The pertinent findings are discussed graphically. 


\section{Mathematical Analysis}

Consider the flow of an electrically conducting, steady two dimensional laminar flow of chemically reacting viscous fluid through porous medium. Magnetic field $B_{0}$ is applied in normal direction to the sheet. The $\mathrm{x}$-axis is along the sheet and $\mathrm{y}$-axis is perpendicular to it. The induced magnetic field is neglected. The governing equations of the motion are:

$$
\begin{gathered}
\frac{\partial u}{\partial x}+\frac{\partial v}{\partial y}=0 \\
u \frac{\partial u}{\partial x}+v \frac{\partial u}{\partial y}=\gamma \frac{\partial^{2} u}{\partial y^{2}}-\frac{\gamma}{K_{0}} u-\frac{\sigma B_{0}^{2}}{\rho} u \\
\rho C_{p}\left[u \frac{\partial T}{\partial x}+v \frac{\partial T}{\partial y}\right]=k^{\prime} \frac{\partial^{2} T}{\partial y^{2}}+\sigma B_{0}{ }^{2} u^{2} \\
{\left[u \frac{\partial C}{\partial x}+v \frac{\partial C}{\partial y}\right]=D_{B} \frac{\partial^{2} C}{\partial y^{2}}-K_{r}\left(C-C_{\infty}\right)}
\end{gathered}
$$

where $u$ and $v$ represent velocity components in $x$ and $y$ directions respectively. $\mu, \rho, \sigma$ and $\gamma=$ $(\mu / \rho)$ represent respectively the coefficient of viscosity, density, electrical conductivity and kinematic viscosity of the fluid. The constant parameters in the system are: $k^{\prime}$ and $C_{p}$ respectively the permeability of porous material and specific heat at constant pressure, $K_{r}$ is the chemical reaction parameter.

The appropriate boundary conditions are,

$$
\begin{aligned}
& u=c x, v=-v_{0} \text { at } \mathrm{y}=0, \quad-k^{\prime}\left[\frac{\partial T}{\partial y}\right]=q_{w}=E_{0} x^{2}, \quad C \rightarrow C_{w} \text { at } y=0 \\
& u \rightarrow 0, \quad T \rightarrow 0, \quad C \rightarrow C_{\infty} \quad \text { as } y \rightarrow \infty
\end{aligned}
$$

\subsection{Solution of the Problem}

In this work, similarity technique is used to solve the system of equations (1)-(4) along with the boundary conditions (5) and (6). The similarity transformations are,

$$
\begin{aligned}
& u=c x f^{\prime}(\eta), v=-\sqrt{\gamma c} f(\eta) \\
& T-T_{\infty}=\frac{E_{o} x^{2}}{k^{\prime}} \theta(\eta), \phi(\eta)=\frac{c-C_{\infty}}{\left(C_{w}-C_{\infty}\right)}
\end{aligned}
$$

where $\eta=y \sqrt{\frac{c}{\gamma}}$ is similarity variable which dimensionless variable. $E_{0}$ is positive constant, $q_{w}$ is the ratio of heat transfer.

Introducing the above transformations in equations (1)-(4). The equation (1) is readily satisfied and the equations (2), (3) and (4) becomes:

$$
\begin{aligned}
& f^{\prime \prime \prime}+f f^{\prime \prime}-f^{2}-\left(M^{2}+\frac{1}{K}\right) f^{\prime}=0, \\
& \theta^{\prime \prime}+\operatorname{Pr} f \theta^{\prime}-2 \operatorname{Pr} f^{\prime} \theta-M^{2} B r f^{\prime 2}=0, \\
& \phi^{\prime \prime}+S c f \phi^{\prime}-S c K r \phi=0 .
\end{aligned}
$$

where $K=\frac{c K_{0}}{\gamma}$ represents the permeability of porous medium, $M^{2}=\frac{\sigma B_{0}^{2}}{\rho c}$ represents the magnetic parameter, $\operatorname{Pr}=\mu C_{p} / \kappa^{\prime}$ represents the Prandtl number, $B r=\frac{\mu C_{p}}{K^{\prime}} \frac{c^{2} x^{2} K^{\prime}}{E_{0} x^{2} C_{p}} \sqrt{\frac{c}{\gamma}}$ denotes the Brinkman number, $K r=\frac{K_{l}}{c}$ is the chemical reaction parameter and $S c$ is the Schmidt number

The boundary conditions (5) and (6) transform to the following form,

$$
\begin{array}{lcl}
f(0)=S, & f^{\prime}(0)= \pm 1, & \theta^{\prime}(0)=-1, \\
f^{\prime}(\infty)=0, & \theta(\infty)=0 & \phi(0)=1, \quad \phi(\infty) \rightarrow 0
\end{array}
$$

Here $f^{\prime \prime}=v, \theta^{\prime}=z, \quad \phi^{\prime}=e^{\prime}$ are the initial guesses which are arbitrarily chosen and an iterative procedure is set to obtain solutions through Runge-Kutta fourth-order method. The computational procedure involved to find the maximum value of $\eta$ for which $f^{\prime}(0) \rightarrow 0, \theta(\eta) \rightarrow 0$ at $\eta \rightarrow \infty$ and $\phi(\eta) \rightarrow 0$ at $\eta \rightarrow \infty$ and find the proper estimates for the unknown quantities $f^{\prime}(0), \theta^{\prime}(0)$ and $\phi^{\prime}(0)$. Their refinement was interpolated iteratively with the prescribed error tolerance. A grid independence study was also carried out to examine the effect of step size $\Delta \eta$. A step size of $\Delta \eta=0.1$ was found to be satisfactory for a convergence criterion of $10^{-7}$ in all cases.

The process is repeated until we obtain the correct results upto the desired accuracy of $10^{-7}$ level.

The non-linear coupled ordinary differential equations (9)-(11) subject to boundary condition

(12) are reduced to a system of first order ordinary differential equations as follows,

$$
\begin{aligned}
& f^{\prime}=w, w^{\prime}=v, v^{\prime}=-x v+(w * w)+\left(M^{2}+\frac{1}{K}\right) w \\
& \theta=y, \theta^{\prime}=z, \phi=b, \phi^{\prime}=e^{\prime}, \quad z^{\prime}=2 \operatorname{Pr} w y-\operatorname{Pr} x z+M^{2} B r w^{2} \\
& \phi=b, \phi^{\prime}=e^{\prime}, e^{\prime}=-S c f e+S c K r z^{\prime}
\end{aligned}
$$


and boundary conditions become,

$x(0)=S, w(0)=1, \quad z(0)=-1 \quad$ at $\quad \eta=0$

$w(0) \rightarrow 0, y(0) \rightarrow 0 \quad$ as $\quad \eta \rightarrow \infty$

3. Results and Discussion

The equations (9) -(11) are solved subject to the boundary conditions (12) are solved by using shooting procedure. The numerical results have been computed by using Mathematica 6.0 software. The effects of the physical parameters namely magnetic parameter $M$, Schmidt number $(S c)$, Chemical reaction $(K r)$ on velocity, temperature and concentration distributions. The results have been presented graphically.

Fig. 1 shows the effect of magnetic parameter $M$ on the velocity profile. It is noticed that the velocity decreases, when magnetic parameter increases. This is because Lorentz's force acts opposite to the flow direction. Temperature profile for various values of magnetic parameter is mentioned in Fig.2. It clearly shows that, temperature increases, when magnetic field increases. The effect of Lorentz force acting on velocity profiles created a kind of friction on the flow, which in turn generated more heat energy which eventually increases the temperature profiles in the flow. Fig. 3 depicts the variation in concentration with respect to magnetic field. Concentration increases numerically with increasing values of magnetic field. This is due to the temperature gradient inherent in the velocity of the fluid.

Fig. 4 shows the effect of Schmidt number on Concentration profile. It shows that, concentration decreases, when Schmidt number increases. Fig. 5 represents the effect of chemical parameter on concentration profile. It is clear that increase in the chemical reaction parameter decreases the concentration profile.

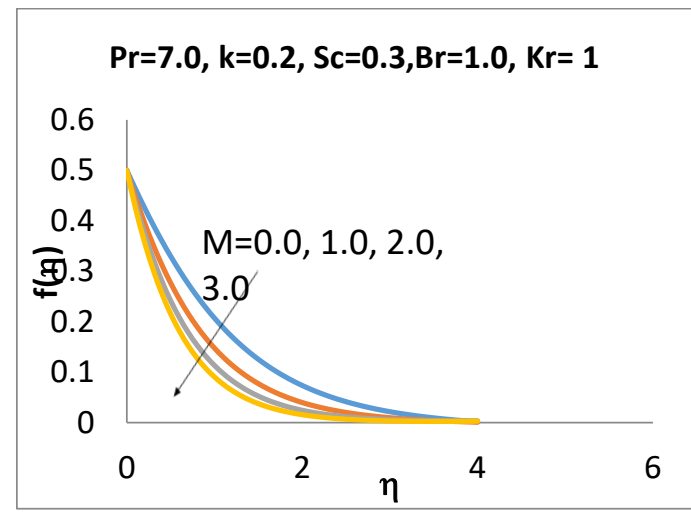

Fig 1 Velocity for various values of Magnetic field $M$

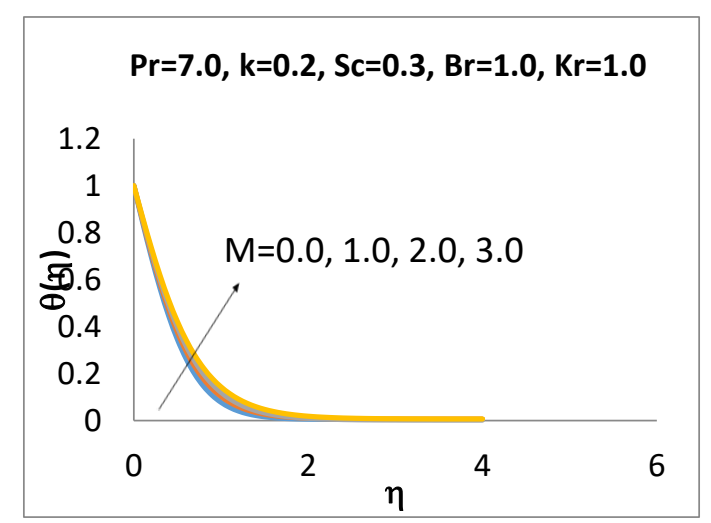

Fig 2 Temperature profile $\theta(\eta)$ for various values of Magnetic field $M$ 


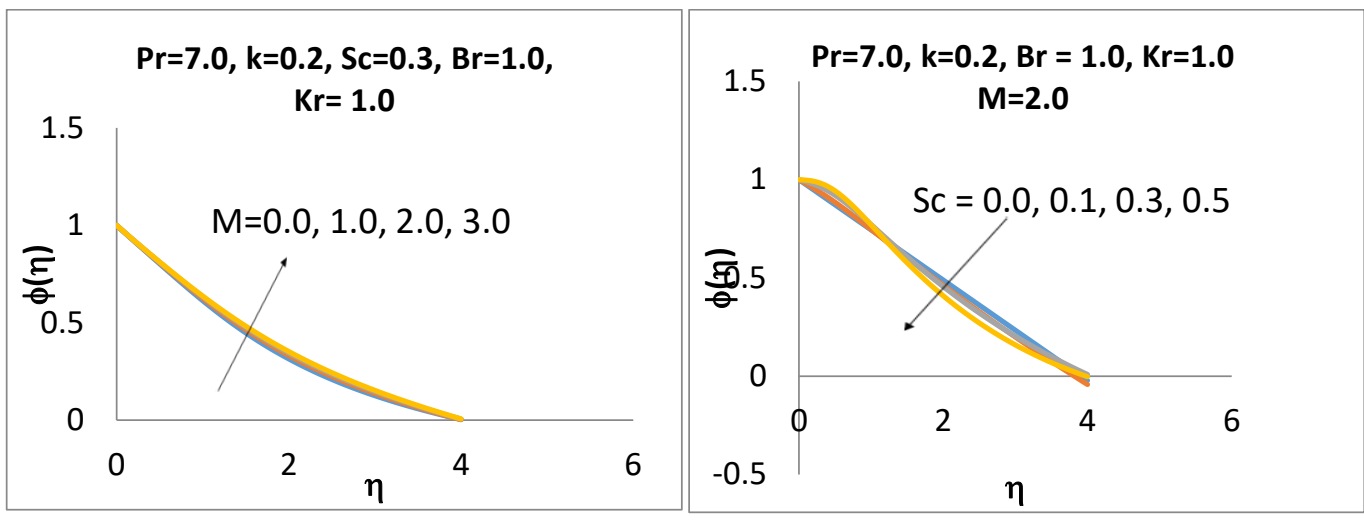

Fig 3Effect of Magnetic field $M$ on

Concentration $\phi(\eta)$

Fig. 4 Concentration profiles $\phi(\eta)$ for various values of $S c$.

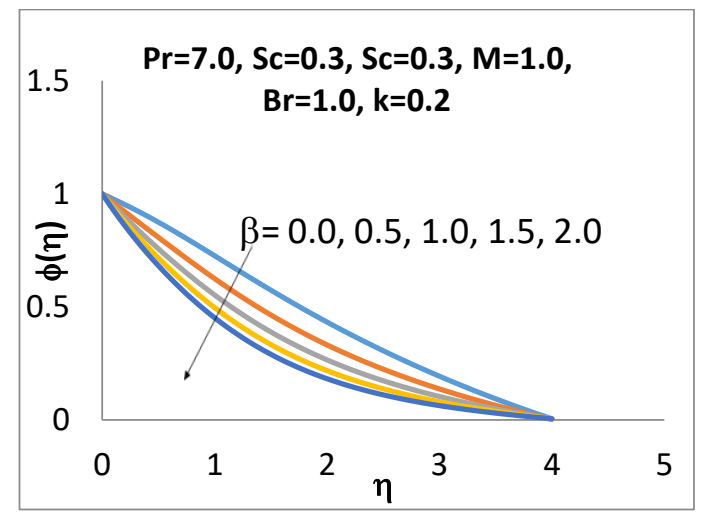

Fig. 5 Effect of chemical parameter on concentration $\phi(\eta)$

\section{Conclusion}

We have investigated the effect of magnetohydrodynamic convective steady flow and heat transfer through a porous medium in the presence of chemical reaction. The Governing equations are solved using shooting procedure with fourth order Runge-Kutta Method - Numerically. The effects of various parameters on the flow and heat transfer are observed from the graphs, and are summarized as follows:

$>$ Velocity profile decreases as we increase the values of magnetic parameter $M$

$>$ Temperature profile increases as we increase the values magnetic parameter $M$

$>$ Concentration profile increases when increase increasing magnetic parameter $M$

$>$ Concentration decreases as we increase the values of Schmidt number and Chemical Parameter

\section{References}

1. [1] H.I. Andersson, Slip flow past a stretching surface, Acta Mechanica, 158, 121-125, 2002.

2. [2] K. Bhattacharyya and I. Pop, MHD boundary layer flow due to an exponentially shrinking sheet, magnetohydrodynamics, 47(4), 337--344, 2011.

3. [3] K. Bhattacharyya and G. C. Layek, Chemically reactive solute distribution in MHD boundary layer flow over a permeable stretching sheet with suction or blowing, Chemical Engineering Communications, 197, 1527-1540, 2010.

4. [4] Bhattacharyya, K. and Layek, G.C., Similarity Solution of MHD Boundary Layer Flow with Diffusion and Chemical Reaction over a Porous Flat Plate with Suction/Blowing, Meccanica, 47, 1043-1048, 2012.

5. [5] Kandasamy R, and Mohamad,R, Radiative heat transfer on nanofluids flow over a porous convective surface in the presence of magnetic field, Journal of Applied Mechanical Engineering, 4, 1-7, 201

6. [6] Mallikarjuna.B, Rashad A.M., Chamkha A.J., Chemical reaction on MHD convective heat and mass transfer flow past a rotating vertical cone embedded in a variable porosity regime, Afr. Mat., 27, 3-4, 2015. 
7. [7] Mohanty.B, Mishra S.R., Pattanayak H.B., Numerical investigation on heat and mass transfer effect of micropolar fluid over a stretching sheet through porous media, Alexandria Engineering Journal, 54, 2, 223-232, 2015.

8. [8] Ullah, I., Khan,I. and Shafie, S, Heat and mass transfer in unsteady MHD slip flow of Casson fluid over a moving wedge embedded in a porous medium in the presence of chemical reaction: Numerical solutions using Keller-Box method, Numerical Methods for Partial Differential Equations, Vol. 2017, 1-25, 2017.

9. [9] Santosh Chaudhary and Pradeep Kumar, MHD Slip Flow past a Shrinking Sheet, Applied Mathematics, 4, 574-581, 2013.

10. [10] Sarma D and Mahanta P.K, Chemical Reaction Effects on a Three Dimensional MHD Mass Transfer Flow past a Vertical Plate, IOSR Journal of Mathematics, 11, 01-13, 2015. 